\title{
Natural Killer (NK) and CAR-NK Cell Expansion Method using Membrane Bound-IL-21-Modified B Cell Line
}

\author{
Minh $\mathrm{Ma}^{1,2}$, Saiaditya Badeti ${ }^{1}$, James K. Kim ${ }^{1}$, Dongfang Liu ${ }^{1,3}$

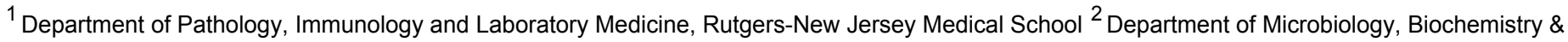

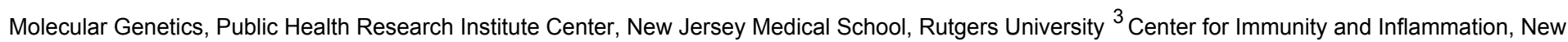 \\ Jersey Medical School, Rutgers-The State University of New Jersey
}

\section{Corresponding Author}

Dongfang Liu

dongfang.liu@rutgers.edu

\section{Citation}

Ma, M., Badeti, S., Kim, J.K.

Liu, D. Natural Killer (NK) and CAR-NK

Cell Expansion Method using Membrane Bound-IL-21-Modified B Cell Line. J. Vis. Exp. (180), e62336, doi:10.3791/62336 (2022).

\section{Date Published}

February 8, 2022

DOI

$10.3791 / 62336$

\section{URL}

jove.com/video/62336

\section{Abstract}

Chimeric antigen receptor (CAR)-modified immune cell therapy has become an emerging treatment for cancers and infectious diseases. NK-based immunotherapy, particularly CAR-NK cell, is one of the most promising 'off-the-shelf' development without severe life-threatening toxicity. However, the bottleneck for developing a successful CAR-NK therapy is achieving sufficient numbers of non-exhaustive, longlived, 'off-the-shelf' CAR-NK cells from a third party. Here, we developed a new CARNK expansion method using an Epstein-Barr virus- (EBV) transformed B cell line expressing a genetically modified membrane form of interleukin-21 (IL-21). In this protocol, step-by-step procedures are provided to expand NK and CAR-NK cells from cord blood and peripheral blood, as well as solid organ tissues. This work will significantly enhance the clinical development of CAR-NK immunotherapy.

\section{Introduction}

Natural killer (NK) cells are an important subset of lymphocytes that express CD56 and lack expression of the $\mathrm{T}$ cell marker, $\mathrm{CD}^{1,2}$. Conventional NK cells are classified as innate immune cells responsible for immunosurveillance of virally infected cells and cancerous cells. Unlike T cells, NK cells recognize infected or malignant cells using CD16 or other activating receptors and do not require the formation of a complex between antigen peptides and major histocompatibility complex (MHC) class I molecules ${ }^{3,4}$.
Recent clinical investigations using chimeric antigen receptor (CAR)-NK cells to treat relapsed or refractory CD19-positive cancers (non-Hodgkin's lymphoma or chronic lymphocytic leukemia [CLL])showed the outstanding safety advantages of CAR-NK cells ${ }^{5}$. For instance, CAR-NK cell infusion is associated with minimal or negligible graft versus host disease (GVHD), neurotoxicity, cardiotoxicity, and cytokine release syndrome $(\mathrm{CRS})^{6,7,8,9,10}$. However, conventional methods to expand human NK cells showed exhaustive 
phenotypes with strong fratricidal killing and telomere shortage, which presents a major challenge in obtaining an adequate number of functional NK cells for adoptive immunotherapy ${ }^{11}$.

To overcome these challenges, a method was developed to expand primary NK cells directly from unfractionated peripheral blood mononuclear cells (PBMCs) or cord blood (CB) using an irradiated and genetically engineered 721.221 (hereinafter, 221) cell line, a human B-lymphoblastoid cell line with low expression of MHC class I molecules ${ }^{3}$. Previous studies showed the importance of IL-21 in NK cell expansion; therefore, a genetically engineered membranebound IL-21 expressing a version of the 721.221 cell line (starting now, 221-mIL-21) was developed $11,12,13,14,15$. The results showed that 221-mIL-21 feeder-cell-expanded primary NK cells were expanded to an average of $>40,000$ fold with persistent high NK cell purity for approximately 2-3 weeks. Additional information regarding the application of this protocol can be found in Yang et al. ${ }^{16}$.

This protocol aims to demonstrate the step-by-step procedure of the novel expansion of PBNK, CBNK, tissue-derived NK, and CAR-NK cells ex vivo.

\section{Protocol}

Human tissues and blood-related work in this protocol follows the guidelines of the Rutgers University Institutional Review Board (IRB)

\section{NK cell expansion from liver tissues (Day 0 ), as shown in Figure 1.}

NOTE: Initial cell number and viability are strongly correlated with the time since organ removal and the initial tissue sample amount. However, if tissues are placed in $30 \mathrm{~mL}$ of Hank's
Balanced Salt Solution (HBSS) and kept on ice or in the fridge at $4{ }^{\circ} \mathrm{C}$ overnight, NK cells can still be expanded at high purity and viability up to $24 \mathrm{~h}$ later.

1. Identify viable tissue areas to obtain lymphocytes from tissues and sections using sterile surgical equipment.

2. Place tissues in $30 \mathrm{~mL}$ of HBSS (w/o calcium or magnesium) and keep on ice until ready to prepare for isolation.

3. Mince the tissue into $<0.5 \mathrm{~cm}$ cubes using sterile razor blades or scissors and forceps inside a biosafety cabinet.

4. Prepare a $1 \mathrm{x}$ collagenase IV solution $(1 \mathrm{mg} / \mathrm{mL})$ by diluting a 10x stock in HBSS (10x Collagenase IV: $10 \mathrm{mg} /$ $\mathrm{mL}$ or $\sim 200 \mathrm{U} / \mathrm{mL}$ ).

5. Place the minced tissue pieces in the tissue dissociator tubes. Fill the tubes with no more than $4 \mathrm{~g}$ of tissue and immerse the tissue pieces in $\sim 10 \mathrm{~mL}$ of $1 \mathrm{x}$ collagenase IV.

NOTE: Using DNase I is not recommended as it may slightly decrease NK viability and yield. Please refer to the Table of Materials for the specific tissue dissociator tubes used.

6. Place the tissue dissociator tubes into a tissue dissociator and blend at $37{ }^{\circ} \mathrm{C}$ to mince the tissue thoroughly.

NOTE: For liver tissue, this may take over $30 \mathrm{~min}$. For more friable tissue, around 15 min may be sufficient. Please refer to the Table of Materials for specific tissue dissociator tubes and the tissue dissociator used.

7. Remove the tissue dissociator tubes and triturate through $40 \mu \mathrm{m}$ nylon cell strainer using the backend of a 5 $\mathrm{mL}$ syringe. Collect the eluent and discard the large undigested fragments. 
8. Spin down the eluent at $400 \times g$ for $5 \mathrm{~min}$ at room temperature. Aspirate the supernatant.

9. Resuspend the cell pellets in $30 \%$ polyvinylpyrrolidone (PVP)-coated silica to remove fat cells that will otherwise contaminate the final lymphocyte fraction.

1. To prepare 1x PVP-coated silica, use 9:1 dilution of PVP-coated silica in 10x PBS.

NOTE: Refer to the Table of Materials for the specific PVP-coated silica used.

2. To prepare $30 \%$ PVP-coated silica, dilute 1x PVPcoated silica with PBS/HBSS.

10. Spin down the cell pellet at $400 \times g$ for $5 \mathrm{~min}$ at room temperature. Aspirate the supernatant.

11. Resuspend the cell pellet in $9 \mathrm{~mL}$ of $\mathrm{R}-10$ media.

NOTE: Refer to the Table of Materials for the composition of R-10 media

12. Carefully layer the cell suspension over $4 \mathrm{~mL}$ of Ficoll or lymphocyte separation media to separate lymphocytes from red blood cells and polymorphonuclear cells.

13. Separate the layers by centrifuging at $400 \times g$ for $23 \mathrm{~min}$ at room temperature with the acceleration and brakes off or at the lowest setting. Carefully decant the uppermedium layer and harvest the interphase containing tissue-infiltrating lymphocytes.

14. Rinse the cells with $10 \mathrm{~mL}$ of media and proceed to cell counting, flow cytometry, aliquoting and freezing of cells, or primary NK expansion protocol.

\section{Primary NK cell expansion from PBMCs (or CB or organ tissues) (Day 0), as shown in Figure 2.}

1. Thaw the frozen PBMC and the frozen, irradiated feeder cells in a $37^{\circ} \mathrm{C}$ water bath.
2. Wash the PBMC and 100 Gamma-irradiated (Gyirradiated) $221-\mathrm{mlL}-21$ cells by centrifugation at $400 \times g$ for 5 min with $10 \mathrm{~mL}$ of $\mathrm{R}-10$ media separately.

3. Save $1 \times 10^{6}$ cells of PBMC for flow cytometry.

NOTE: The initial NK cell purity is an important factor in calculating NK cell expansion rate.

4. Resuspend the cells in $1 \mathrm{~mL}$ of $\mathrm{R}-10$ media. Count the cells using Trypan Blue.

5. Mix $5 \times 10^{6}$ cells of PBMC with $10 \times 10^{6}$ cells of 100 Gyirradiated 221-mIL-21 cells in a special 6-well plate (see Table of Materials).

6. Add $30 \mathrm{~mL}$ of $\mathrm{R}-10$ media supplemented with human IL-2 (200 U/mL) and human IL-15 (5 ng/mL) (see Table of Materials).

7. Incubate the special 6 -well plate at $37{ }^{\circ} \mathrm{C}$ with $5 \% \mathrm{CO}_{2}$.

8. Replace the media with $\mathrm{R}-10$ media supplemented with human IL-2 (200 U/mL) and human IL-15 (5 ng/mL) to maintain NK cells every 3-4 days.

NOTE: Maintain less than $20 \times 10^{6}$ cells per well for further expansion at each media change. For the best viability, ensure that the total cell number in each well does not exceed $100 \times 10^{6}$ cells.

9. Record the total cell number, viability and perform flow cytometry every 3-4 days to calculate the NK cell expansion rate.

\section{Attachment of 293T cells (Day 2), as shown in} Figure 2.

1. Split $1.8 \times 10^{6} 293 \mathrm{~T}$ cells in $11 \mathrm{~mL}$ of $\mathrm{D}-10$ media per treated $100 \mathrm{~mm}$ plate.

NOTE: Refer to the Table of Materials for the composition of $\mathrm{D}-10$ media 
2. Incubate $293 \mathrm{~T}$ cells at $37^{\circ} \mathrm{C}$ with $5 \% \mathrm{CO}_{2}$.

\section{Retrovirus transfection (Day 3)}

1. In a $1.7 \mathrm{~mL}$ tube, mix $470 \mu \mathrm{L}$ of reduced serum media with $30 \mu \mathrm{L}$ of transfection reagent.

NOTE: Refer to the Table of Materials for the specific reduced serum media and transfection reagent used.

2. In a separate $1.7 \mathrm{~mL}$ tube, add $2.5 \mu \mathrm{g}$ of pRDF plasmid, $3.75 \mu \mathrm{g}$ of Pegpam3 plasmid, and $2.5 \mu \mathrm{g}$ of CAR construct in SFG vector into the reduced serum media so that the final volume is $500 \mu \mathrm{L}$.

3. Mix the solutions in steps 4.1 and 4.2 dropwise.

4. Incubate the tube at room temperature for $15 \mathrm{~min}$.

5. Add $1 \mathrm{~mL}$ of the mixture from step 4.4 to $293 \mathrm{~T}$ cell plate on Day 1 in a dropwise manner.

6. Incubate the plate(s) at $37^{\circ} \mathrm{C}$ with $5 \% \mathrm{CO}_{2}$ for $48-72 \mathrm{~h}$.

\section{Retronectin plate-coating (Day 3)}

1. Dilute retronectin protein with Phosphate Buffered Saline (PBS) to a final concentration of $50-100 \mu \mathrm{g} / \mathrm{mL}$.

2. Add $500 \mu \mathrm{L}$ of the diluted retronectin into each well of an untreated 24 -well plate ( 5 wells per CAR construct). Seal the plate using parafilm and incubate the plate at $4{ }^{\circ} \mathrm{C}$ overnight.

\section{Transduction (Day 4)}

1. Centrifuge the retronectin plate at $2103 \times g$ for $30 \mathrm{~min}$ at $4{ }^{\circ} \mathrm{C}$. Discard the supernatant.

2. Block each well of the 24 -well plate with $1 \mathrm{~mL}$ of $\mathrm{R}-10$ medium.

3. Incubate the plate at $37{ }^{\circ} \mathrm{C}$ with $5 \% \mathrm{CO}_{2}$ for $1 \mathrm{~h}$.
4. Pre-warm the centrifuge to $32{ }^{\circ} \mathrm{C}$ while the retronectin plate is being blocked.

5. Collect the retrovirus supernatant by filtering the transfected 293T cells using a $0.45 \mu \mathrm{m}$ filter.

6. Aliquot $2 \mathrm{~mL}$ of the filtered retrovirus supernatant into each well.

7. Centrifuge the 24 -well plate at $2103 \times g$ for $2 \mathrm{~h}$ at $32^{\circ} \mathrm{C}$.

8. During plate centrifugation, collect the expanded PBNK cells from Day 0 and count the cells using Trypan Blue.

NOTE: Continue expanding PBNK cells by adding R-10 media supplemented with IL-2 (200 U/mL) and IL-15 (5 $\mathrm{ng} / \mathrm{mL})$.

9. Dilute the expanded PBNK cells with R-10 media supplemented with IL-2 (200 U/mL) and IL-15 (5 ng/mL) to $2.5 \times 10^{5}-5 \times 10^{5}$ cells $/ \mathrm{mL}\left(0.5 \times 10^{6}-1 \times 10^{6}\right.$ cells per well).

NOTE: Record the total cell number, viability, and save $5 \times 10^{5}$ expanded PBNK cells for flow cytometry as these values are important in determining the NK cell expansion rate.

10. After centrifugation, partially aspirate the retrovirus supernatant from each well.

NOTE: Do not completely aspirate, i.e., leave approximately $100 \mu \mathrm{L}$ of retrovirus supernatant per well, as this will decrease the transduction efficiency.

11. Aliquot $2 \mathrm{~mL}$ of the diluted expanded PBNK cells from step 6.8 to each well.

12. Centrifuge the plate at $600 \times g$ for $10 \mathrm{~min}$ at $32{ }^{\circ} \mathrm{C}$. Incubate the plate at $37^{\circ} \mathrm{C}$ with $5 \% \mathrm{CO}_{2}$ for $48-72 \mathrm{~h}$. NOTE: Do not parafilm the plate. 


\section{CAR-NK cells collection (Day 6 or 7 ), as shown in Figure 2.}

1. Gently collect the cells from the 24-well plate and transfer the cells to a $50 \mathrm{~mL}$ centrifuge tube

NOTE: Try not to generate bubbles, as this will result in a decrease in cell viability.

2. Centrifuge the tube at $400 \times g$ for $5 \mathrm{~min}$.

3. Resuspend the pellet with $1 \mathrm{~mL}$ of $\mathrm{R}-10$ media and count the cells using Trypan Blue.

NOTE: Save $5 \times 10^{5}$ cells for flow cytometry to determine the transduction efficiency.

4. Transfer the resuspended cells to a special 6-well plate containing $30 \mathrm{~mL}$ of R-10 media supplemented with IL-2 (200 U/mL) and IL-15 (5 ng/mL).

5. Incubate the special 6 -well plate at $37{ }^{\circ} \mathrm{C}$ with $5 \% \mathrm{CO}_{2}$.

6. Replace R-10 media supplemented with IL-2 $(200 \mathrm{U} / \mathrm{mL})$ and IL-15 $(5 \mathrm{ng} / \mathrm{mL})$ to maintain NK cells every 3 - 4 days. NOTE: Maintain less than $20 \times 10^{6}$ cells per well for further expansion at each change. For the best viability, ensure that the total cell number in each well does not exceed $100 \times 10^{6}$ cells.

7. Record the total cell number, viability, and perform flow cytometry every 3-4 days to calculate the NK cell expansion rate.

8. Use the cells for appropriate in vitro or in vivo assays. NOTE: The ex vivo expanded PBNK, and CAR-NK cells can be cultured in a $37^{\circ} \mathrm{C}$ incubator for approximately 4 weeks.

9. Examine the NK cell number and purity at day 7, day 11, day 14 , day 18 , and day 21 by flow cytometry.

\section{Representative Results}

A schematic workflow of tissue-infiltrating NK cell isolation and PBNK cell expansion using the 221-mIL-21 feeder cell methodology is shown in Figure $\mathbf{1}$ and Figure 2. Expanded PBNK cells were collected every 3 or 4 days for flow cytometry to determine the NK cell purity by staining cells with anti-human CD56 and anti-human CD3. The experiment was repeated using two different donors to show the reproducibility of the expansion system (Figure 3). PBNK cells expanded by 221-mIL-21 were shown to expand nearly $5 \times 10^{4}$ folds (Figure 3A). Furthermore, the NK cell purity was highly maintained, around $85 \%$ throughout the 21-day expansion (Figure 3B). Using the 221-mIL-21 feeder cell expansion system, the NK cell purity consistently ranged between $85 \%-95 \%$, independent of the donors (data not shown). To demonstrate the robustness of the 221-mIL-21 expansion system, PBMCs were stained for anti-CD56 and anti-CD3 prior to the expansion, which showed a cell purity of $7.09 \%$ for NK cells and a high percentage of $T$ cells (Figure 4A). PBMCs were cocultured with 221-mIL-21 to expand NK cells; the NK purity was checked prior to CAR-NK transduction on Day 4 (Figure 4A). CAR-NK cells were collected and stained for antiCD56, anti-CD3, and anti-hlgG(H+L) $F\left(a b^{\prime}\right) 2$, which showed a high NK cell population $(86.9 \%$ on Day 7$)$ and a high CAR transduction efficiency of approximately $70 \%$ (Figure 4). Higher transduction efficiencies (up to 95\%) were also observed using the retrovirus packaging system. Altogether, these data show that the 221-mIL-21 feeder cells could successfully expand NK cells and preserve the NK cell purity ex vivo. 


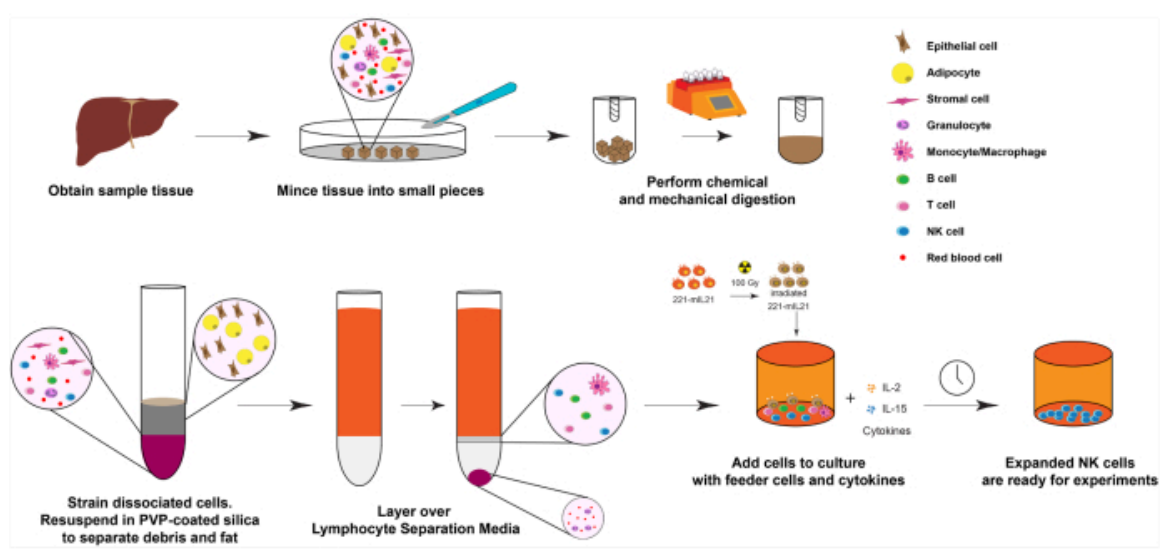

Figure 1: Diagram of NK cell expansion from solid human organ samples. Briefly, the obtained human liver samples are minced into small cubes for mechanical digestion. Dissociated cells are then isolated using PVP-coated silica and Lymphocyte Separation Media. Further, the NK cells are expanded using the expansion protocol described in Figure 2. Please click here to view a larger version of this figure. 

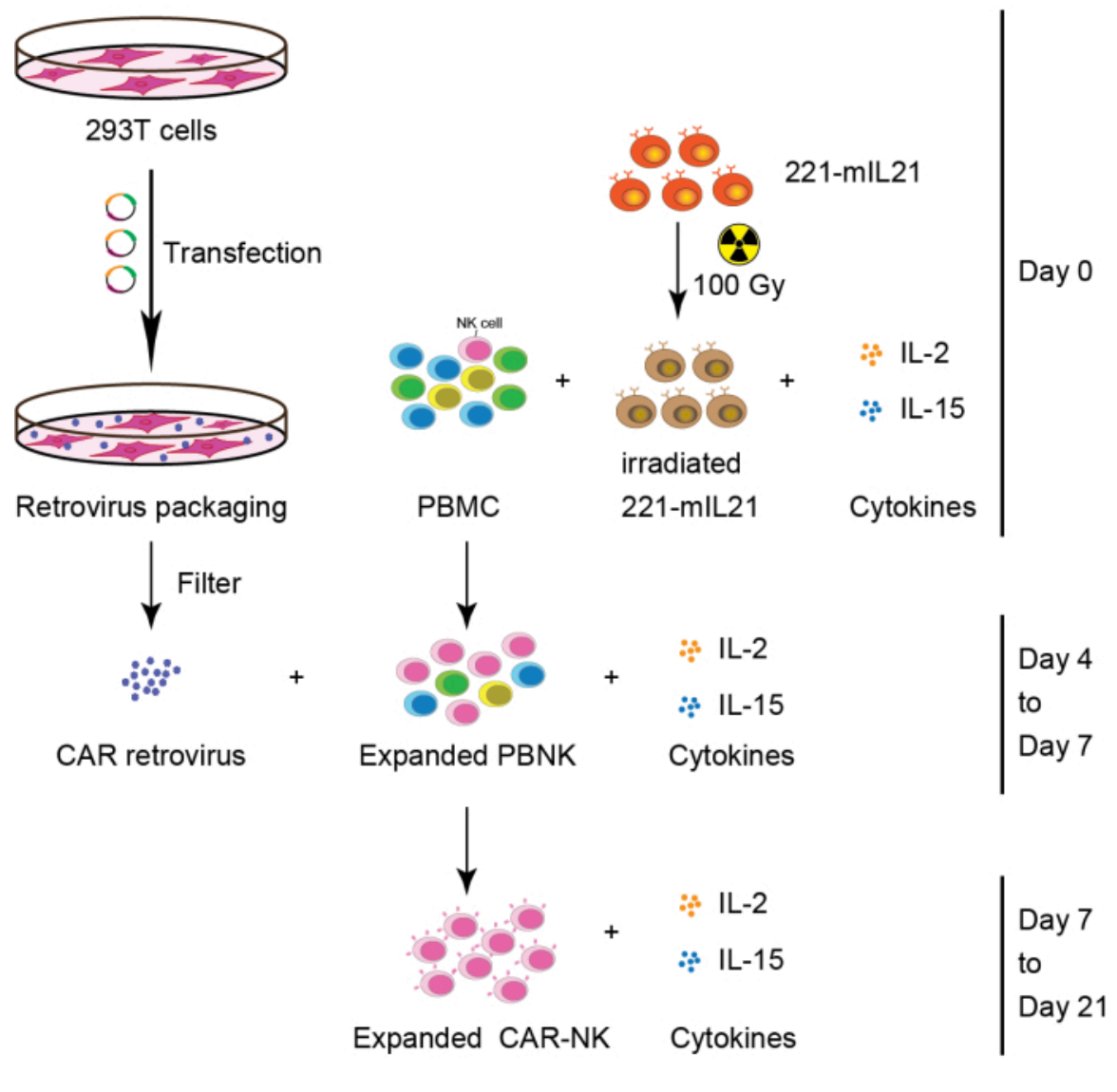

Retrovirus packaging

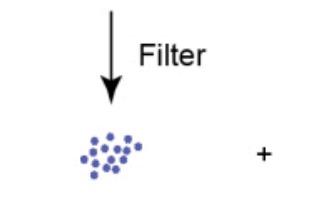

CAR retrovirus

Expa

Figure 2: Schematic workflow of CAR-NK cell generation from PBMCs. Briefly, 221-mIL21 feeder cells were irradiated at 100 Gy prior to coculturing with PBMCs supplemented with IL-2 and IL-15 on Day 0. In parallel, 293T cells were transfected with the retrovirus packaging system to produce CAR retrovirus that was then transduced into the expanded PBNK cells in the presence of IL-2 and IL-15. Primary CAR-NK cells were harvested on Day 7 and continued expansion for 21 days. This figure has been modified from Yang et al. ${ }^{16}$. Please click here to view a larger version of this figure. 
A

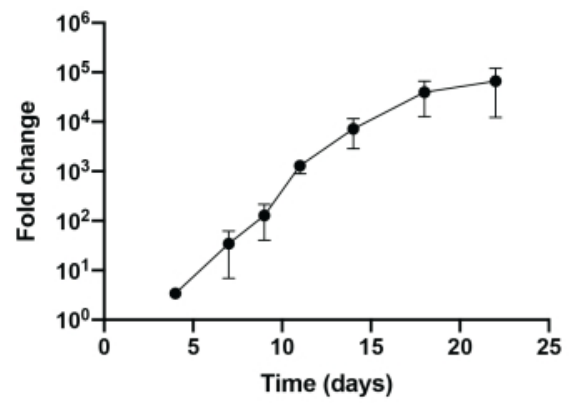

B

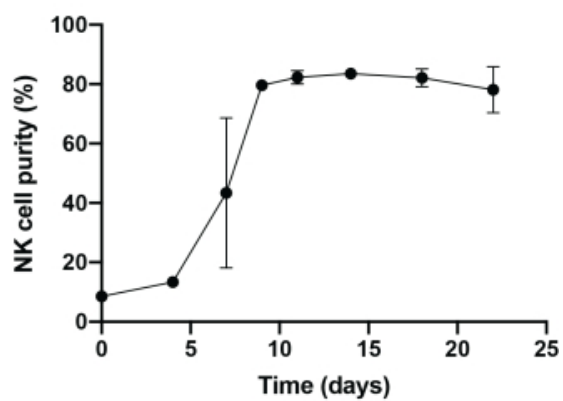

Figure 3: Dynamic time-lapsed expansion of PBNK cells. (A) Fold expansion of PBNK during a 22- day time course. Cells were stained with anti-CD56 and anti-CD3 at indicated days for flow cytometry. The total number of NK cells was determined by multiplying NK cell purity to the total number of cells. Expansion rate was generated as follows: (Number of NK cells) $\mathrm{Tn} /($ Number of NK cells $) \mathrm{T0}$, where Number of NK cells $=$ (percentage of NK cell purity) $\times$ (total number of cells), $\mathrm{T}_{0}$ is the number of NK cells at time day 0 , and Tn is the number of NK cells at time day $n$. (B) NK cell purity during a 22-day time course. The NK cell expansion was repeated two times with two different donors. Error bars represent \pm SEM. Please click here to view a larger version of this figure. 

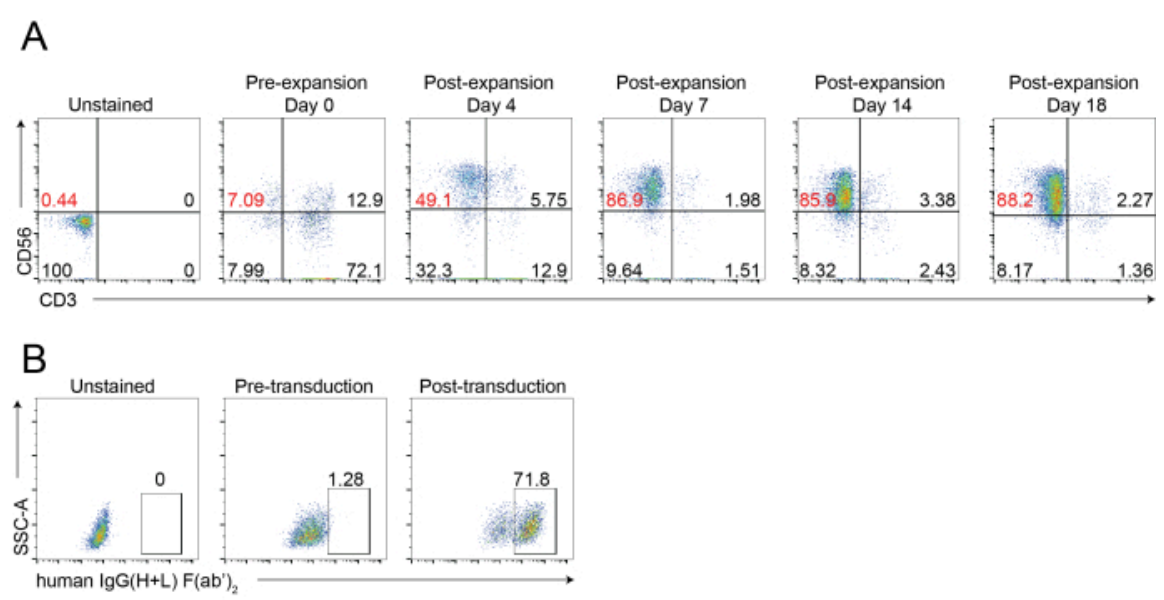

C

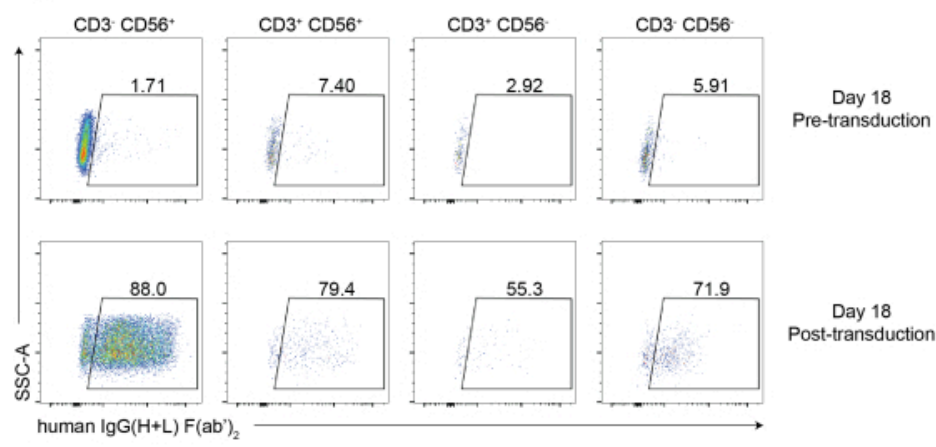

Figure 4: Representative flow cytometric analysis of CAR-NK cells. (A) Representative dot plots showing the dynamic time-lapse of NK cell purity of the CAR-NK cells during an 18-day course. Flow cytometry analysis was assessed by staining the cells with anti-CD56 and anti-CD3 at indicated timepoints. Day 0 indicates pre-expansion of PBNK. Day 4 indicates post-expansion of PBNK and pre-transduction of CAR-NK cells. Day 7 indicates the post-transduction of CAR-NK cells. (B) Representative dot plots showing the transduction efficiency of CAR-NK cells using the retrovirus packaging system. Cells were stained with anti-CD56, anti-CD3, and anti-hlgG(H+L) F(ab')2 for flow cytometry. (C) Representative dot plots showing the CAR expression in various subsets, including $\mathrm{CD}^{2} 6^{+} \mathrm{CD}^{-}, \mathrm{CD}^{-} 6^{-} \mathrm{CD} 3^{+}, \mathrm{CD} 6^{+} \mathrm{CD}^{+}$, and $\mathrm{CD}^{-} 6^{-} \mathrm{CD} 3^{-}$on Day $18 . \mathrm{Cells}^{-}$ were stained with anti-CD56, anti-CD3, and anti-hlgG(H+L) F(ab')2 (indicating CAR expression) for flow cytometry. Please click here to view a larger version of this figure.

\section{Discussion}

Most of the current CAR-NK products in clinical trials utilize NK cell lines ${ }^{17}$, such as NK-92, a cell line isolated from a non-Hodgkin's lymphoma patient ${ }^{18}$, NK-92MI, IL-2 independent NK-92 cell line ${ }^{19}$, and NKL, isolated from a large granular lymphocyte patient ${ }^{20}$, as these cell lines are easily proliferative for 'off-the-shelf' products. However, these cell lines, e.g., NK-92 cells, have marginal clinical efficacies and in vivo expansion, as they require irradiation prior to infusion, thus limiting their proliferation and cytotoxicity in vivo 21 . 
Given these reasons, various strategies are currently being explored to expand primary NK cells from several sources, including peripheral blood, $\mathrm{CB}$, bone marrow (BM), human embryonic stem cells (HSCs), induced pluripotent stem cells (iPSCs), and tumor tissues $21,22,23$. For instance, NK cells can be expanded ex vivo using interleukins including IL-15, IL-18, and IL-21. Lymphoblastoid cell lines such as K562 cells or Epstein-Barr Virus-transformed lymphoblastoid cell lines such as 721.221 cells, are also used for NK cell expansion $^{16}$. However, the aforementioned strategies often generate insufficient number of NK cells for an adoptive transfer of CAR-NK immunotherapy 22,24 . To help solve the problem, the study here shows a protocol for an ex vivo NK cell expansion using a genetically modified EBV-transformed cell line, 221-mIL-21 feeder cells.

The expansion methodology using 221-mIL-21 feeder cells shown in this protocol is optimized to expand NK cells with an expansion rate of at least 10 to 100-fold higher than other leukemia cell lines, including $\mathrm{HL}-60$ and $\mathrm{OCl}-$ AML3 expressing membrane IL-21, K562, and K652-mIL21 expressing OX40 ligand $22,24,25$. The CAR expression is also evaluated for approximately 2 weeks ex vivo. More significantly, the 221-mIL-21 feeder cell expansion strategy can be applied to expand NK cells from various sources, including PBMCs, CB, and solid organs such as the liver, without an initial NK enrichment step. Although the 221mIL-21 feeder system is not as donor-dependent as the aforementioned feeder cell lines, it is not entirely independent of donors. On average, the 221-mIL-21 expansion system can achieve $90 \%$ of NK cell purity with a high NK cell number, with approximately $<5 \%$ of $\mathrm{T}$ cell contamination on day 14 post-expansion. Therefore, to eliminate the possibilities of $\mathrm{T}$ cell contamination, it is necessary to isolate NK cells from obtained samples prior to the ex vivo expansion or use a
$\mathrm{CD}^{+}$selection system to eliminate $\mathrm{T}$ cells after an ex vivo expansion.

One of the criticisms in using an NK cell expansion system is that the feeder cells may not have been fully eradicated after the expansion or prior to a transfusion, which may possess significant regulatory concerns; therefore, complete eradication of feeder cells before a transfusion is crucial. However, recent CAR-NK clinical trials in which K562-mIL21-4-1BBL feeder cells were used for the ex vivo CBNK cell expansion ${ }^{24,25}$ showed no concerning complications. Furthermore, our preliminary data showed a gradual decrease of the irradiated $221-\mathrm{mlL}-21$ population as the expansion progressed (data not shown). However, more extensive studies are required for this expansion method to be implemented in a clinical setting. Collectively, the 221-mIL-21 expansion system helps solve the challenge of expanding primary CAR-NK cells, and therefore will significantly contribute to the broader use of CAR-NK cellbased immunotherapy in the near future.

\section{Disclosures}

The authors declare no competing interests.

\section{Acknowledgments}

We would like to thank the members of the Liu laboratory (Dr. Hsiang-chi Tseng, Dr. Xuening Wang, and Dr. ChihHsiung Chen) for their comments on the manuscripts. We would like to thank Dr. Gianpietro Dotti for the SFG vectors and Dr. Eric Long for the 721.221 cells. This work was supported in part from HL125018 (D. Liu), Al124769 (D. Liu), Al129594 (D. Liu), Al130197 (D. Liu), and Rutgers-Health Advance Funding (NIH REACH program), U01HL150852 (R. Panettieri, S. Libutti, and R. Pasqualini), S10OD025182 (D. 
Liu), and Rutgers University-New Jersey Medical School Startup funding for D. Liu Laboratory.

\section{References}

1. Van Acker, H. H. et al. CD56 in the immune system: More than a marker for cytotoxicity? Frontiers in Immunology. 8, 892 (2017).

2. Caligiuri, M. A. Human natural killer cells. Blood. 112 (3), 461-469 (2008).

3. Shimizu, Y. et al. Transfer and expression of three cloned human non-HLA-A,B,C class I major histocompatibility complex genes in mutant lymphoblastoid cells. Proceedings of the Nationall Academy of Sciences of the United States of America. 85 (1), 227-231 (1988).

4. Wu, J., Lanier. L. L. Natural killer cells and cancer. Advances in Cancer Research. 90, 127-156 (2003).

5. Liu, E. et al. Use of CAR-transduced natural killer cells in CD19-positive lymphoid tumors. The New England Journal of Medicine. 382 (6), 545-553 (2020).

6. Alonso-Camino, V. et al. Efficacy and toxicity management of CAR-T-cell immunotherapy: a matter of responsiveness control or tumour-specificity? Biochemical Society Transactions. 44 (2), 406-411 (2016).

7. Bonifant, C. L., Jackson, H. J., Brentjens, R. J., Curran, K. J. Toxicity and management in CAR T-cell therapy. Molecular Therapy Oncolytics. 3, 16011 (2016).

8. Kalaitsidou, M., Kueberuwa, G., Schitt, A., Gilham, D. E. CAR T-cell therapy: toxicity and the relevance of preclinical models. Immunotherapy. 7 (5), 487-97 (2015).

9. Gust, J. et al. Endothelial activation and bloodbrain barrier disruption in neurotoxicity after adoptive immunotherapy with CD19 CAR-T cells. Cancer Discovery. 7 (12), 1404-1419 (2017).

10. Hay, K. A. et al. Kinetics and biomarkers of severe cytokine release syndrome after CD19 chimeric antigen receptor-modified T-cell therapy. Blood. 130 (21), 2295-2306 (2017).

11. Zhang, Y. et al. In vivo kinetics of human natural killer cells: the effects of ageing and acute and chronic viral infection. Immunology. 121 (2),258-265 (2007).

12. Vidard, L. et al. CD137 (4-1BB) Engagement fine-tunes synergistic IL-15- and IL-21-driven nk cell proliferation. Journal of Immunology (Baltimore, Md.:1950). 203 (3), 676-685 (2019).

13. Venkatasubramanian, S. et al. IL-21-dependent expansion of memory-like NK cells enhances protective immune responses against Mycobacterium tuberculosis. Mucosal Immunology. 10 (4), 1031-1042 (2017).

14. Ojo, E. O. et al. Membrane bound IL-21 based NK cell feeder cells drive robust expansion and metabolic activation of NK cells. Scientific Reports. 9 (1), 14916 (2019).

15. Denman, C. J. et al. Membrane-bound IL-21 promotes sustained ex vivo proliferation of human natural killer cells. PLoS One. 7 (1), e30264 (2012).

16. Yang, Y. et al. Superior expansion and cytotoxicity of human primary NK and CAR-NK cells from various sources via enriched metabolic pathways. Molecular Therapy. Methods \& Clinical Development. 18, 428-445 (2020).

17. Liu, S. et al. NK cell-based cancer immunotherapy: from basic biology to clinical development. Journal of Hematology \& Oncology. 14 (1), 7 (2021). 
18. Gong, J. H., G. Maki, Klingemann, H. G. Characterization of a human cell-line (Nk-92) with phenotypical and functional-characteristics of activated natural-killer-cells. Leukemia. 8 (4), 652-658 (1994).

19. Tam, Y. K. et al. Characterization of genetically altered, interleukin 2-independent natural killer cell lines suitable for adoptive cellular immunotherapy. Human Gene Therapy. 10 (8), 1359-1373 (1999).

20. Robertson, M. J. et al. Characterization of a cell line, NKL, derived from an aggressive human natural killer cell leukemia. Experimental Hematology. 24 (3), 406-415 (1996).

21. Hu, Y., Tian, Z. G., Zhang, C. Chimeric antigen receptor (CAR)-transduced natural killer cells in tumor immunotherapy. Acta Pharmacologica Sinica. 39 (2), 167-176 (2018).

22. Tseng, H. C. et al. Efficacy of anti-CD147 chimeric antigen receptors targeting hepatocellular carcinoma. Nature Communications. 11 (1), 4810 (2020).

23. Easom, N. J. W. et al. IL-15 overcomes hepatocellular carcinoma-induced NK cell dysfunction. Frontiers in Immunology. 9, 1009 (2018).

24. Granzin, M. et al. Highly efficient IL-21 and feeder cell-driven ex vivo expansion of human NK cells with therapeutic activity in a xenograft mouse model of melanoma. Oncoimmunology. 5 (9), e1219007 (2016).

25. Liu, E. L. et al. Cord blood derived natural killer cells engineered with a chimeric antigen receptor targeting CD19 and expressing IL-15 have long term persistence and exert potent anti-leukemia activity. Blood. 126 (23) (2015). 